\title{
Preliminary study of synthetic aperture tissue harmonic imaging on in-vivo data
}

Rasmussen, Joachim Hee; Hemmsen, Martin Christian; Sloth Madsen, Signe ; Møller Hansen, Peter; Bachmann Nielsen, Michael ; Jensen, Jørgen Arendt

\section{Published in:}

Proceedings of SPIE

Link to article, DOI:

$10.1117 / 12.2006363$

Publication date:

2013

Link back to DTU Orbit

Citation (APA):

Rasmussen, J. H., Hemmsen, M. C., Sloth Madsen, S., Møller Hansen, P., Bachmann Nielsen, M., \& Jensen, J. A. (2013). Preliminary study of synthetic aperture tissue harmonic imaging on in-vivo data. In Proceedings of SPIE: Medical Imaging 2013: Ultrasonic Imaging, Tomography, and Therapy (Vol. 8675). SPIE - International Society for Optical Engineering. https://doi.org/10.1117/12.2006363

\section{General rights}

Copyright and moral rights for the publications made accessible in the public portal are retained by the authors and/or other copyright owners and it is a condition of accessing publications that users recognise and abide by the legal requirements associated with these rights.

- Users may download and print one copy of any publication from the public portal for the purpose of private study or research.

- You may not further distribute the material or use it for any profit-making activity or commercial gain

- You may freely distribute the URL identifying the publication in the public portal 


\title{
Preliminary study of synthetic aperture tissue harmonic imaging on in-vivo data
}

\author{
Joachim Hee Rasmussen $^{a}$, Martin Christian Hemmsen ${ }^{a}$, Signe Sloth Madsen ${ }^{b}$, Peter Møller \\ Hansen $^{b}$, Michael Bachmann Nielsen ${ }^{b}$ and Jørgen Arendt Jensen ${ }^{a}$ \\ ${ }^{a}$ Center for Fast Ultrasound Imaging, Technical University of Denmark, 2800 Lyngby, Denmark \\ ${ }^{b}$ Department of Radiology, Copenhagen University Hospital, Rigshospitalet, 2100 Copenhagen, \\ Denmark
}

\begin{abstract}
A method for synthetic aperture tissue harmonic imaging is investigated. It combines synthetic aperture sequential beamforming (SASB) with tissue harmonic imaging (THI) to produce an increased and more uniform spatial resolution and improved side lobe reduction compared to conventional B-mode imaging. Synthetic aperture sequential beamforming tissue harmonic imaging (SASB-THI) was implemented on a commercially available BK 2202 Pro Focus UltraView ultrasound system and compared to dynamic receive focused tissue harmonic imaging (DRF-THI) in clinical scans. The scan sequence that was implemented on the UltraView system acquires both SASB-THI and DRF-THI simultaneously. Twenty-four simultaneously acquired video sequences of in-vivo abdominal SASB-THI and DRF-THI scans on 3 volunteers of 4 different sections of liver and kidney tissues were created. Videos of the in-vivo scans were presented in double blinded studies to two radiologists for image quality performance scoring. Limitations to the systems transmit stage prevented user defined transmit apodization to be applied. Field II simulations showed that side lobes in SASB could be improved by using Hanning transmit apodization. Results from the image quality study show, that in the current configuration on the UltraView system, where no transmit apodization was applied, SASB-THI and DRF-THI produced equally good images. It is expected that given the use of transmit apodization, SASB-THI could be further improved.
\end{abstract}

Keywords: Non-linear imaging, Second harmonic imaging, Tissue harmonic imaging, Synthetic aperture imaging, Virtual sources, In-vivo evalutaion

\section{INTRODUCTION}

Tissue harmonic imaging (THI) has been combined with a variety of imaging techniques to further improve image quality. Tissue harmonic imaging (THI) benefits from improved spatial resolution, lower side lobe levels, less reverberation, and a more narrow beam profile compared to regular B-mode imaging. ${ }^{1,2}$ As a transmitted ultrasound waveform travels through tissues, the waveform gets distorted by the nonlinear properties of the different tissues. These non-linear properties cause the speed of sound in the tissues to change due to the difference in acoustic pressure in the peaks and troughs of the transmitted waveform. ${ }^{3}$ This causes the high pressure peaks to travel faster than the relatively low pressure troughs. As the waveform gets distorted, higher harmonics are generated in the waveform. The strength of these harmonics is dependent on the strength of the emitted ultrasound field. It is therefore important to emit a field intense enough to ensure good development of harmonic waveforms.

One approach to obtain tissue harmonic imaging (THI) is by using a pulse inversion scheme. ${ }^{4,5}$ Here, two identical, but individually phase inverted excitation pulses, are transmitted for every line in the B-mode image. By summing the responses, the second harmonic waveform is enhanced, while neighboring harmonic components (the fundamental and third harmonic waveforms) are attenuated.

THI has long been combined with various focusing techniques, including dynamic receive focusing (DRF) to further improve image quality. Synthetic aperture imaging (SAI) is a focusing technique that generates focused

Further author information: Joachim Hee Rasmussen: E-mail: jr@elektro.dtu.dk, Telephone: (+45) 45253902 
images in both transmit and receive. ${ }^{6-8}$ In the synthetic aperture focusing technique (SAFT) ${ }^{9}$ an active aperture of a single element is used in transmit and receive. For every position of the active aperture, a low resolution image is constructed and in the end all low resolution images are combined to create one high resolution image. Naturally, because only one element is used, the signal-to-noise ratio (SNR) is very poor using this technique. Also, because a full low resolution image has to be beamformed for every line, the computational load and beamforming is extensive. Several SAI techniques have been suggested to improve SNR, including the use of multi-element transmit and receive aperture as suggested by Karaman et al. ${ }^{10}$ Here a group of elements are used in transmit to increase the SNR. By focusing multiple elements in a single point in transmit, a virtual source is created as first described by Passmann and Ermert ${ }^{11}$ and further investigated by Frazier and O'Brien, ${ }^{12}$ Nikolov and Jensen, ${ }^{13,14}$ and Bae and Jeong. ${ }^{15}$

The use of several electronically focused elements in the aperture for generating virtual sources causes the ultrasound energy field to be focused. This causes development of higher harmonic ultrasound waveforms as described in THI. Depending on the magnitude of the emitted field, a tissue harmonic image using SAI could be generated by isolating and imaging the second harmonic waveform of the received response. Such a technique was first suggested by Bae et $\mathrm{al}^{16}$ in 2008 to generate a fully focused, high resolution image. Still, the major drawback, however, is the computational load required to produce SAI.

Synthetic aperture sequential beamforming (SASB) is a technique that utilizes a two step beamformer to significantly reduce computational processing load compared to traditional SAI. ${ }^{17,18}$ In SASB, a set of image lines are first obtained and beamformed using a fixed focus in both transmit and receive. Secondly, the image data are beamformed using the fixed focus points as virtual sources to obtain the final synthetic aperture image. The advantage of SASB, besides the reduction in computational load, is that the lateral resolution remains higher and more uniform over depth compared to regular dynamic receive focusing (DRF). ${ }^{19}$ The use of virtual sources in SASB has been shown to create an acoustic field intense enough to generate harmonics for THI. ${ }^{20,21}$

The purpose of this study is to implement SASB-THI on a commercial system and optimize the technique for clinical imaging. Furthermore, the purpose is to show if there is a difference between SASB-THI and DRF-THI on in-vivo image data acquired simultaneously for the two techniques. Finally, the purpose of this study is to show how SASB-THI on the commercial system can be further improved by use of transmit apodization.

\section{METHODS}

First stage SASB images can be generated using basically any ultrasound system capable of fixed transmit and receive focusing. If the ultrasound system is furthermore capable of pulse inversion, the system may be used to generate first stage SASB-THI. Instead of having only one emission for every fixed focus SASB line in the image, the two phase inverted excitation pulses are emitted for the same line position with the same fixed focus. The two pulses should be emitted interchangeably to minimize tissue movement between the two responses, which would compromise the final summed image.

\subsection{Implementation on commercial ultrasound system}

A commercially available BK 2202 Pro Focus UltraView (BK-Medical, Herlev, Denmark) ultrasound scanner was used for scanning. The scan sequence performed both SASB-THI and DRF-THI simultaneously. On the UltraView scanner, a DRF-THI setup is the default imaging technique, when performing abdominal scans. All settings for DRF-THI have been optimized for clinical scans by the manufacturer, which makes DRF-THI the obvious candidate for a golden standard to evaluate SASB-THI against. The DRF-THI scheme implemented in this case also uses the pulse inversion technique to obtain the second harmonic component for imaging.

The scan sequence was designed to minimize time delay between the pairs of excitation waveforms (regular and phase inverted waveforms). Each frame consisted of both a SASB-THI frame and a DRF-THI frame. First, 
the SASB-THI frame was acquired, where for each image line, the waveform pairs were transmitted interchangeably. Secondly, the DRF-THI frame was acquired in much the same manner with transmit of the regular and phase inverted waveforms interleaved. In this manner, tissue motion between the regular and phase inverted excitation waveforms was kept at a minimum. Any tissue motion would effectively compromise the success of second harmonic waveform enhancement and separation using pulse inversion. The whole scan sequence was then repeated to acquire a collection of frames. An illustration showing the scan sequence on the UltraView system is shown in Fig. 1A.

A

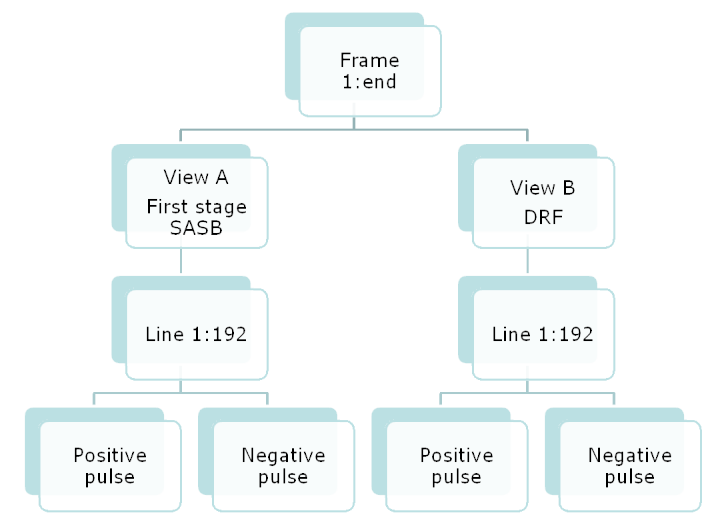

B

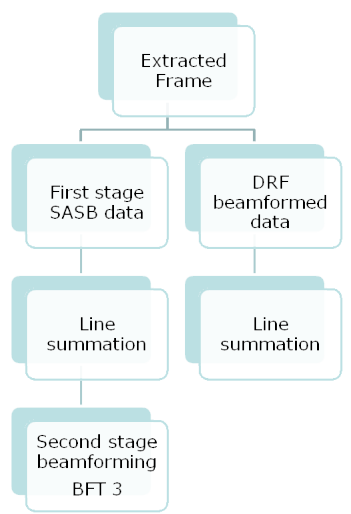

Figure 1. Graphical visualization of $\mathbf{A}$ ) the scan sequence on the UltraView system and $\mathbf{B}$ ) post processing steps.

The scanner was equipped with a UA2227 research interface (BK-Medical) that allows first stage image data to be acquired from the scanner and stored on a PC. For SASB-THI a 64 element fixed aperture with focus at $80 \mathrm{~mm}$ was used in transmit and receive with an F-number of 3. For DRF-THI a 64 element fixed aperture with focus of $85 \mathrm{~mm}$ and an F-number of 5.3 was used in transmit, while a 64 dynamic receive aperture and an F-number of 0.8 was used in receive. Limitations in the scanner's transmit stage prevent user defined transmit apodization to be applied, and so for both SASB-THI and DRF-THI the default transmit apodization was a 64 element boxcar. This can affect the quality of the final image as artifacts such as e.g. side lobes are more pronounced when no apodization is used. In receive, however, apodization is possible. Here, a Gauss window was applied for both SASB-THI and DRF-THI.

A one-and-a-half cycle excitation pulse with a center frequency of $2.14 \mathrm{MHz}$ and its phase inverted copy were used for both SASB-THI and DRF-THI. The scanner was fitted with a 192 element convex array transducer (BK 8820e, BK-Medical) commonly used in abdominal tissue harmonic scans. This transducer has a center frequency of 3.5 MHz and recommended operational frequency range of 2-6 MHz. This ensured that transmission of the $2.14 \mathrm{MHz}$ excitation pulse and reception of the $4.28 \mathrm{MHz}$ second harmonic response both were well within the operational range of the transducer. The scanner was connected via a X64-CL Express camera link (Dalsa, Waterloo, Ontario, Canada) to a PC. Data were acquired on the PC using an acquisition routine that was run in MATLAB (MathWorks, Natick, MA, USA).

Abdominal in-vivo scans were made on 3 healthy volunteers by radiologists from Copenhagen University Hospital, Rigshospitalet. Four scan positions of liver and kidney tissues were imaged twice for each volunteer. The scan procedure was as follows. The scan sequence was loaded and initialized on the scanner and the data acquisition was initialized in MATLAB on the data acquisition PC. The radiologist found a good view of liver or kidney tissues on one of the scan locations. A sequence of 50 frames was then acquired from the scanner and stored on the PC. Pulse summation was performed on the pairs of corresponding first stage image lines and the acquired first stage SASB-THI data were beamformed using the BFT3 toolbox ${ }^{22}$ to produce second stage image 
data. Both the second stage SASB-THI data and DRF-THI data were then stored and compiled into video sequence files, which were also stored. The pulse summation, beamforming, video compiling, and data storing were performed using BFT3 in a MATLAB routine. An illustration showing the post processing steps for data extraction and beamforming is shown in Fig. 1B.

The performance of SASB-THI was evaluated against DRF-THI by two radiologists who were presented to the two video sequences made from each scan. The video sequences were presented in the program IQap, ${ }^{23}$ which automatically and randomly selected two corresponding video files from the collection of scans and presented the videos on screen next to each other. The program randomly selected on which side of the screen to present each imaging technique. The viewing radiologist did therefore not know which scan was being watched and which imaging technique was presented on either side of the screen. The radiologist was asked to score which of the shown sequences was the best based on spatial resolution, image contrast, noise, and unwanted artifacts on a visual analog scale (VAS). The VAS was in the range of -50 to 50 corresponding to left view is best - left view is slightly better - no difference - right view slightly better - right view is best. An example of how a video sequence was presented to the radiologist is shown in Fig. 2.

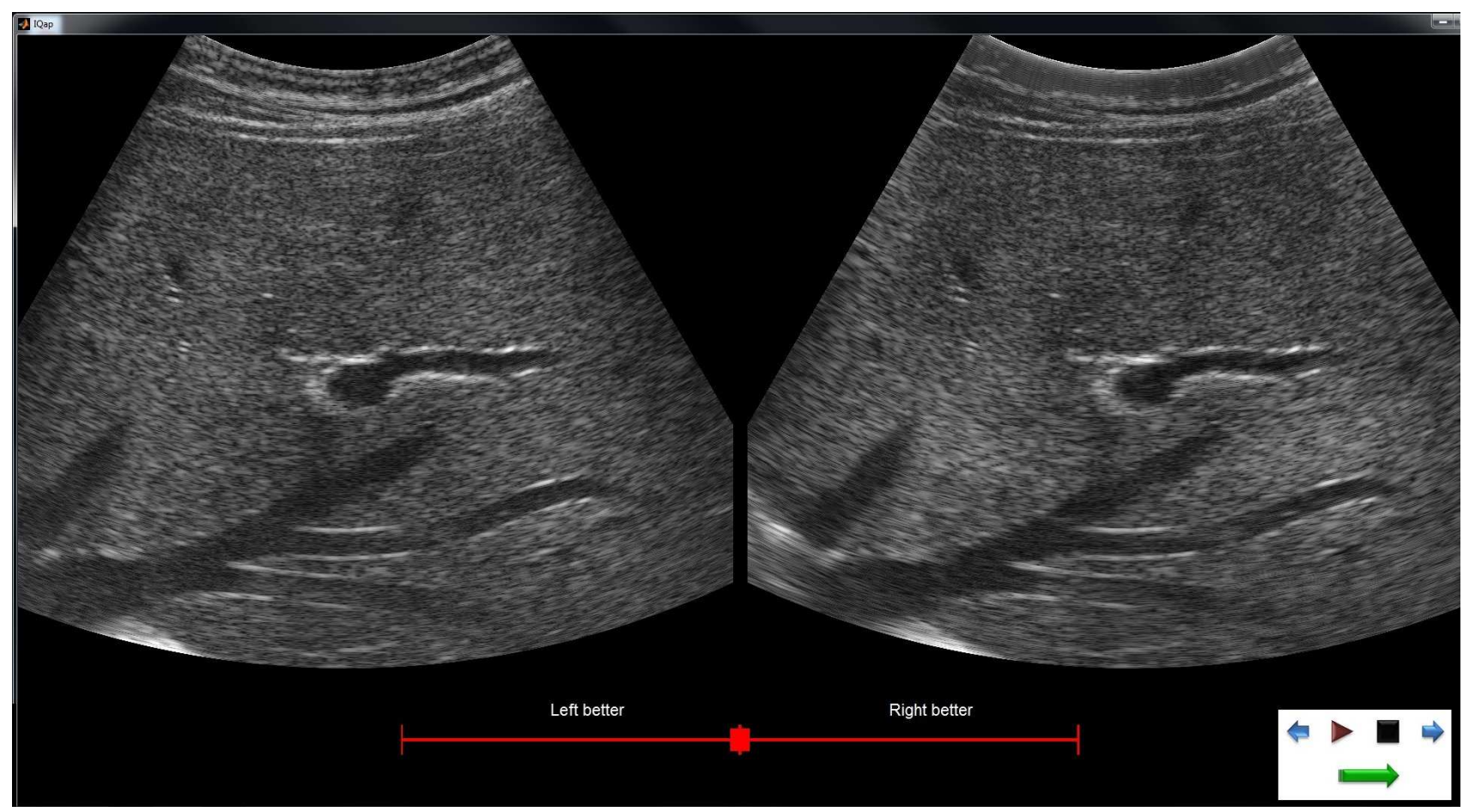

Figure 2. Visualization of an image pair for overall image quality evaluation. The VAS is shown in the bottom and the control panel for navigating the sequences are shown in the lower right corner. In this case, DRF-THI is shown on the left and SASB-THI is shown on the right.

\section{RESULTS}

A total of 24 sets of videos from $2 \times 4$ scans on 3 volunteers were made using SASB-THI and DRF-THI. Examples of images using the two techniques are shown in Fig. 3 and Fig. 4 . Note, that in all examples in Fig. 3 and Fig. 4 the scan geometry is the same using the two techniques. This makes comparison of the two techniques much more straightforward as differences in scan geometry do not need to be taken into account. Fig $3 \mathrm{~A}$ and Fig. 3B show three large arteries through the liver imaged on two different volunteers. Fig 4A and Fig. 4B show a section of a kidney plus a lobe of the liver from the same two volunteers. In all cases SASB-THI is shown on the left and DRF-THI on the right. 

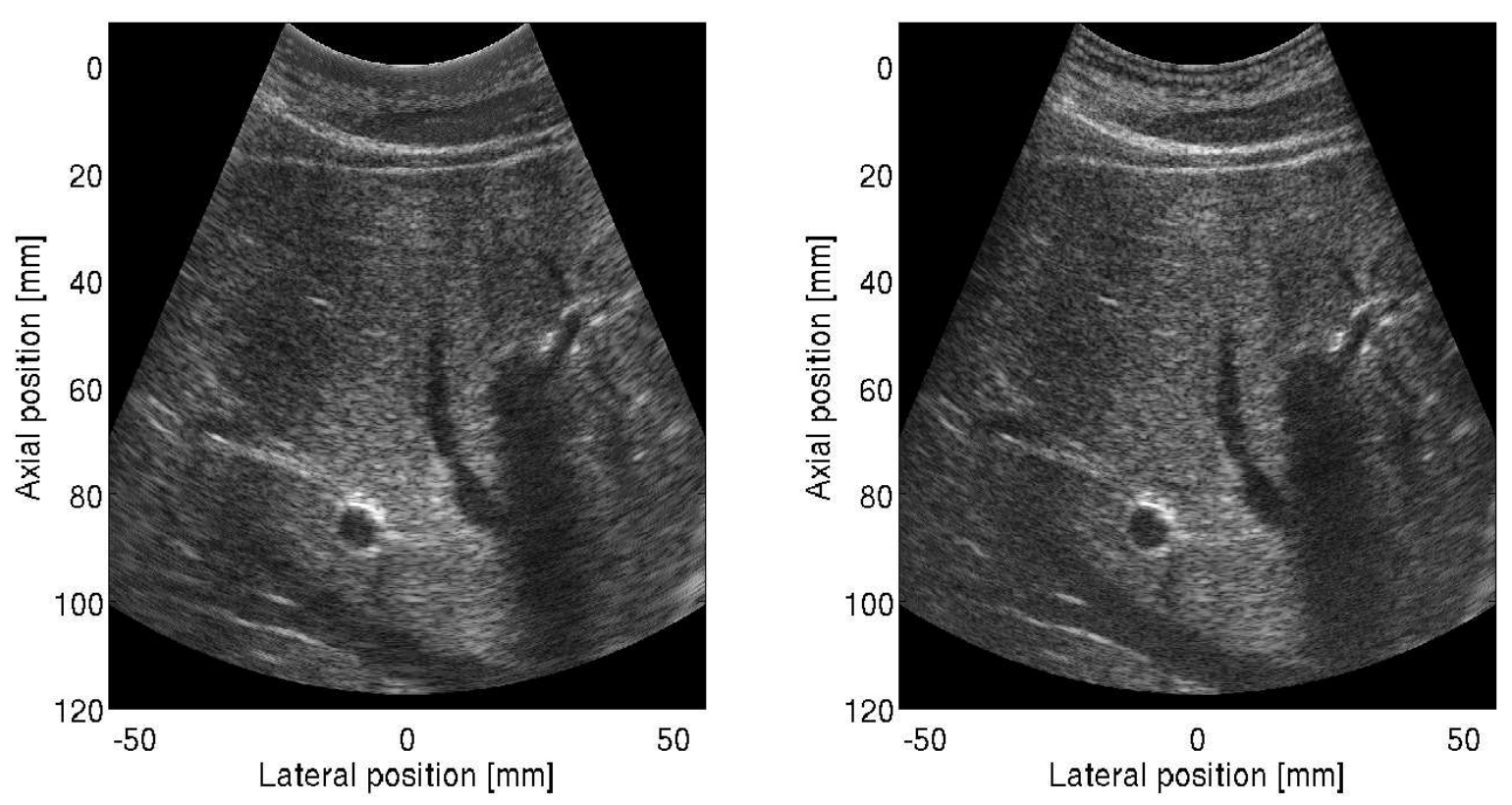

B
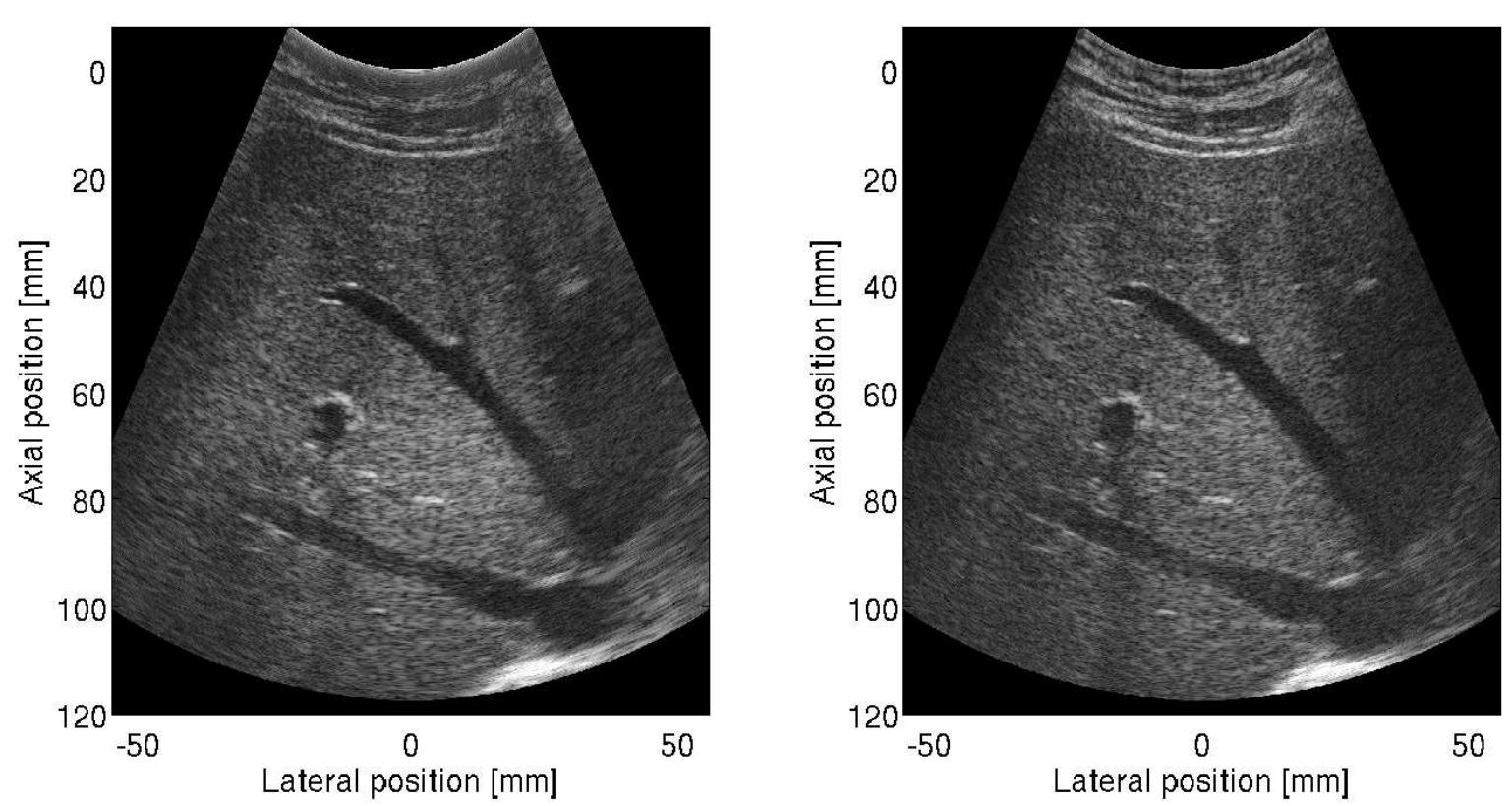

Figure 3. Clinical images acquired using the scan sequence on the UltraView system. Images show both scan techniques (SASB-THI and DRF-THI) from one scan location (liver) on two different volunteers. In both cases SASB-THI is shown on the left and DRF-THI on the right. 

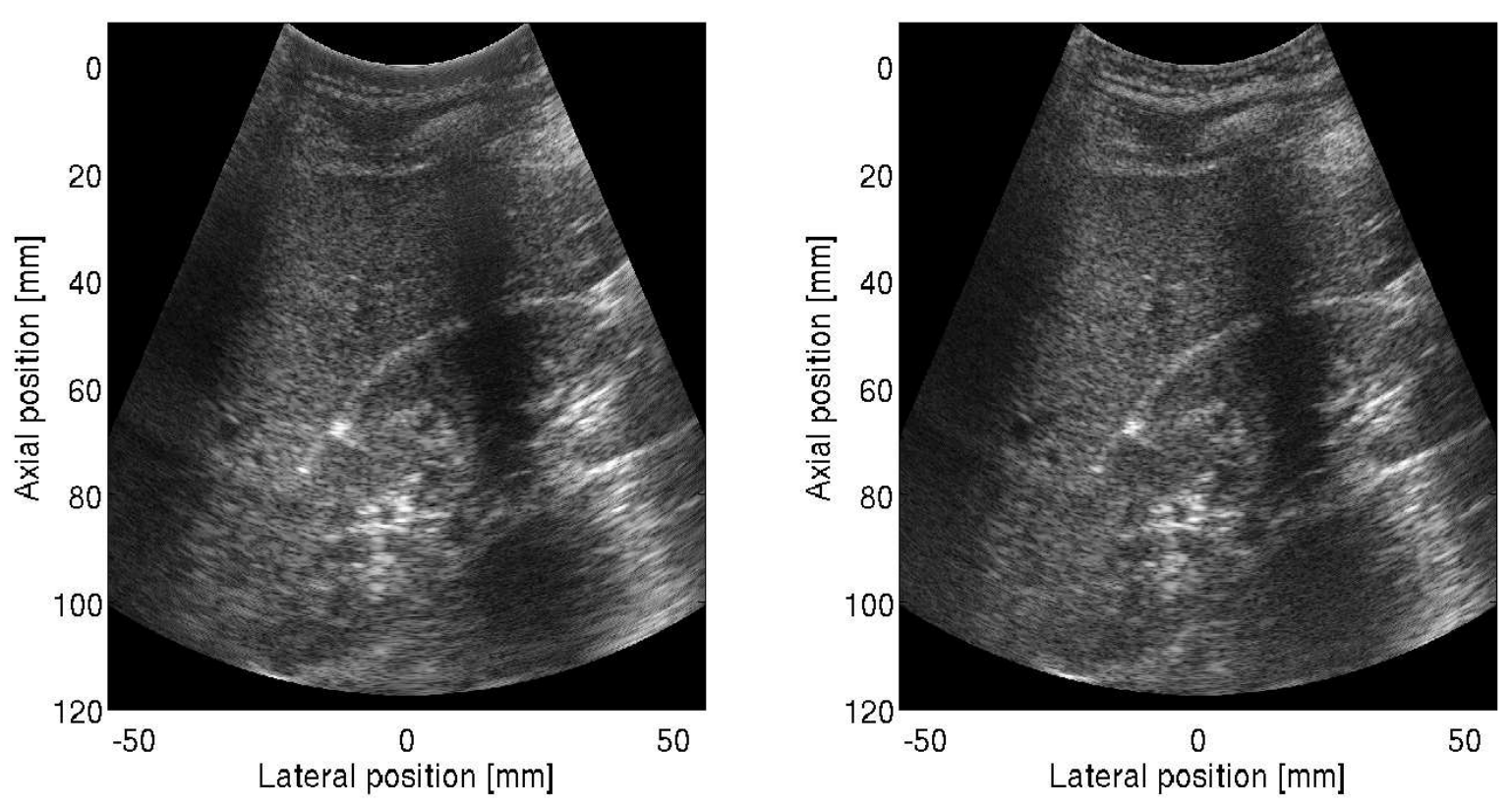

B
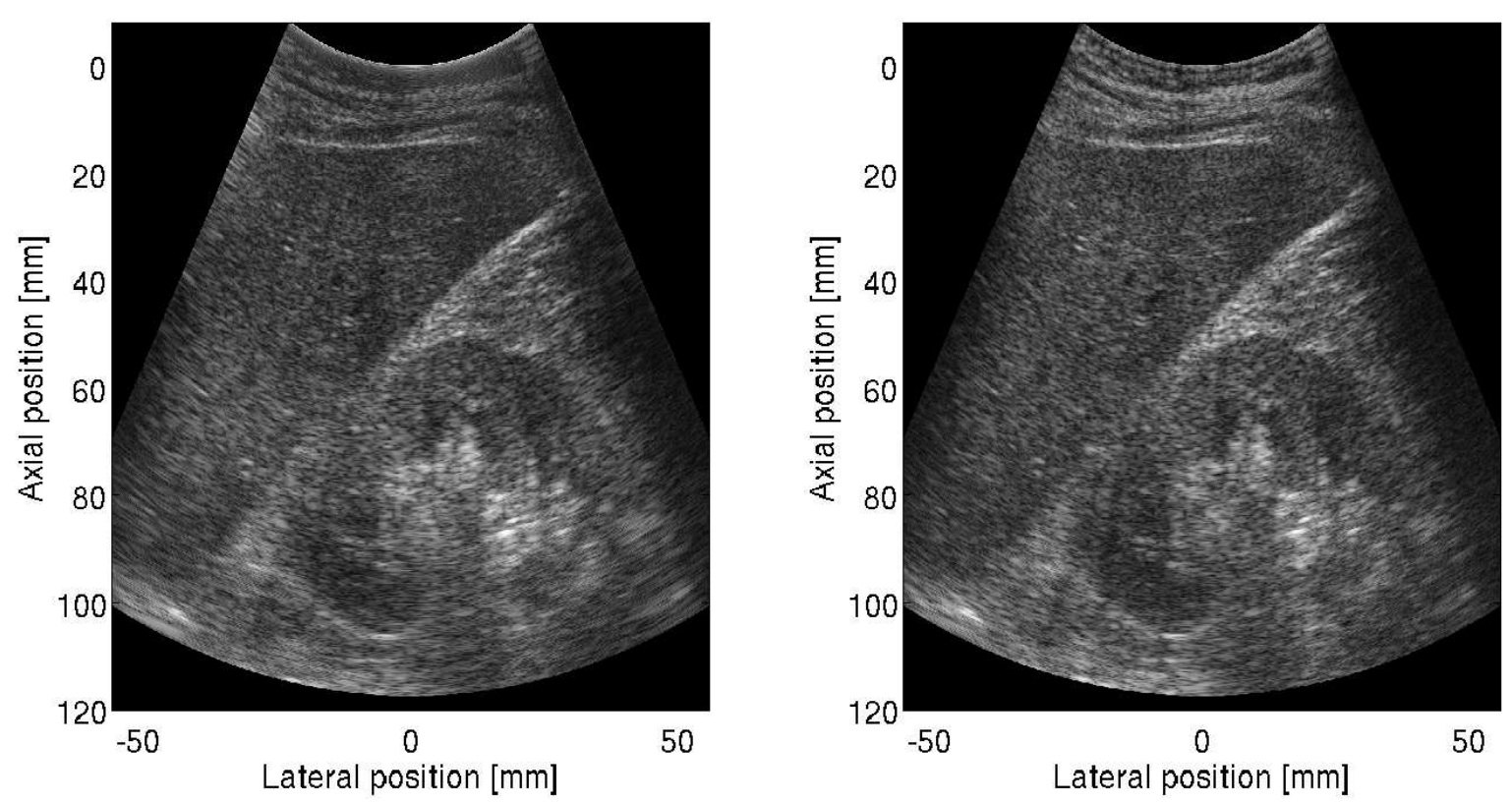

Figure 4. Clinical images acquired using the scan sequence on the UltraView system. Images show both scan techniques (SASB-THI and DRF-THI) from one scan location (kidney and liver lobe) on two different volunteers. In both cases SASB-THI is shown on the left and DRF-THI on the right. 
It is seen from Fig. 3 and Fig. 4 that there is very little difference in the image quality in the two scan techniques. Both resolution and contrast in the two images from all scans are almost identical. Visual artifacts from e.g. ribs are present and are equally pronounced in both images in Fig. 3A, Fig. 3B, and Fig. 4A. Penetration depth is also the same for the two techniques.

The video sequences were presented to the two radiologist (A and B) twice in a double blinded study for scoring and the VAS scores were collected. In both cases, a positive VAS score favors DRF-THI over SASB-THI meaning DRF-THI produced better images compared to SASB-THI.

The histograms of the VAS scores from both radiologists are shown in Figure 5. The accumulated median of all VAS scores from radiologist $\mathrm{A}$ was +6 with the $25 \%$ and $75 \%$ quantiles at +5 and +9 . The accumulated median of all VAS scores from radiologist B was +2 with the $25 \%$ and $75 \%$ quantiles at 0 and +4 . These results show that the radiologists found the two techniques to be very similar in terms of image quality.
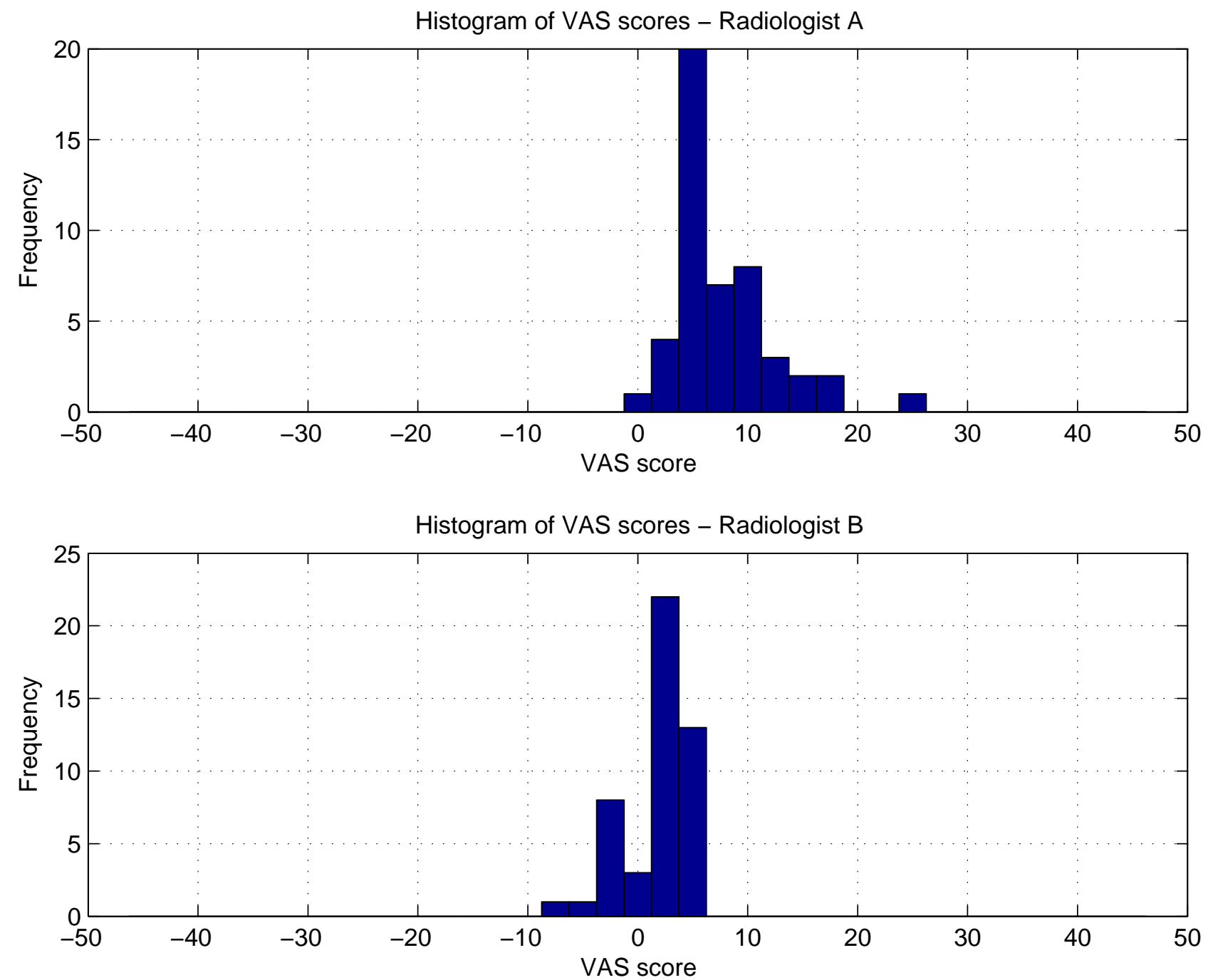

Figure 5. Histogram of VAS scores from Radiologist A and B. A negative VAS score indicates SASB-THI was preferred, a positive VAS score that DRF-THI was preferred. VAS scores are in the interval -50 to 50 corresponding to left view is best - left view is slightly better - no difference - right view slightly better - right view is best. 


\section{DISCUSSION AND CONCLUSION}

A synthetic aperture tissue harmonic imaging technique was implemented successfully on a commercially available ultrasound system. The technique was used to perform in-vivo clinical investigations and was compared to DRF-THI, which is the common imaging technique in abdominal investigations. Since SASB-THI data were acquired simultaneously with DRF-THI data, variations in positions of the transducer during scans was excluded and therefore allowed for a fair comparison of the two techniques. DRF-THI was chosen as a golden standard to compare SASB-THI against since DRF-THI on the commercial system was already optimized for in-vivo scans by the manufacturer.

Visual comparison of the two scan techniques showed only very little differences in terms of resolution, contrast and penetration depth. Visual artifacts such as shadowing were present using both techniques, but were equally pronounced. Videos of the in-vivo scans performed on volunteers were shown to and scored by two radiologists in a double blinded study to exclude bias. Statistical investigations showed that the radiologists found that both techniques performed close to equally good in terms of image quality.

The first stage beamformer in SASB is of very low complexity as it only requires one delay profile. The second stage beamformer applies the output lines from the first stage beamformer rather than channel rf-data and dynamically focuses the image. This way of beamforming reduces the amount of computational calculations compared to traditional SAI and DRF imaging.

The results suggest, that in the scan sequence on the UltraView system, SASB-THI can produce images that are as good as DRF-THI, while reducing the amount of computational calculations.

The transmit stage on the UltraView system did not permit application of transmit apodization. It is hypothesized, that improvements to the reduction of side lobes in SASB-THI could be made by applying user defined transmit apodization. Fig. 6 show contour plots of the point spread functions for SASB simulation using Field $\mathrm{II}^{24,25}$ with either boxcar transmit apodization or Hanning transmit apodization. Here, it is seen how transmit apodization can reduce the level of side lobes in SASB. It is expected that the improvements in side lobes for SASB also apply for SASB-THI. The limitations to the transmit stage on UltraView suggests that further studies using a system capable of transmit apodization should be made on in-vivo image data. It is expected, that the application of transmit apodization will further improve the image quality of clinical images using SASB-THI.

\section{ACKNOWLEDGMENTS}

This work was supported by grant 024-2008-3 from the Danish Advanced Technology Foundation and BK Medical Aps, Denmark

\section{REFERENCES}

[1] B. Ward, A. C. Baker, and V. F. Humphrey, "Nonlinear propagation applied to the improvement of resolution in diagnostoc medical ultrasound," J. Acoust. Soc. Am. 101(1), pp. 143-154, 1997.

[2] T. Christopher, "Finite amplitude distortion-based inhomogeneous pulse echo ultrasonic imaging," IEEE Trans. Ultrason., Ferroelec., Freq. Contr. 44(1), pp. 125-139, 1997.

[3] R. T. Beyer, Nonlinear Acoustics, Department of the Navy, 1974.

[4] C. S. Chapman and J. C. Lazenby, "Ultrasound imaging system employing phase inversion subtraction to enhance the image," US Patent 5632277, 1997.

[5] D. H. Simpson, C. T. Chin, and P. N. Burns, "Pulse inversion Doppler: a new method for detecting nonlinear echoes from microbubble contrast agents," IEEE Trans. Ultrason., Ferroelec., Freq. Contr. 46(2), pp. 372-382, 1999 . 

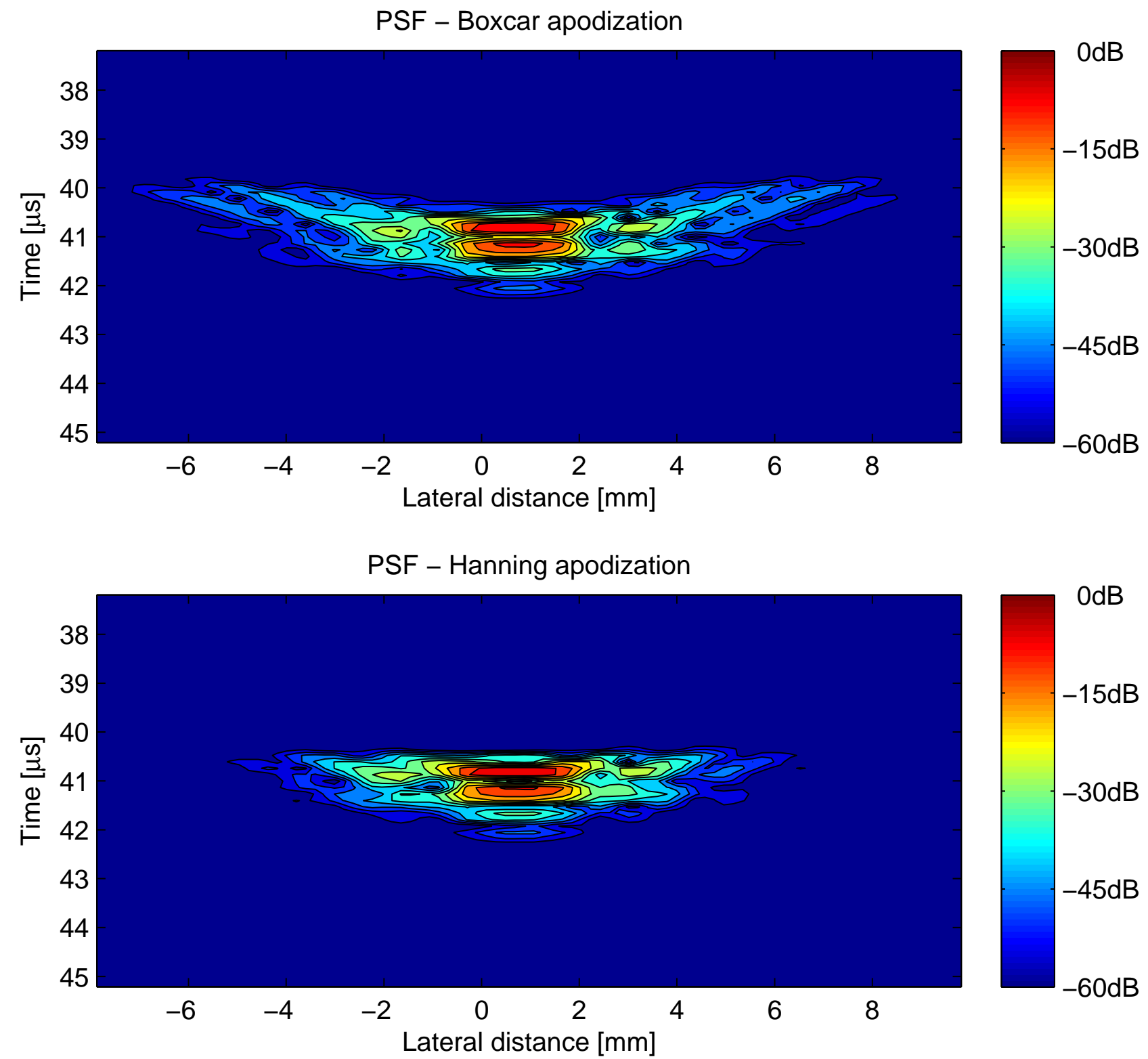

Figure 6. Simulated point spread functions for SASB using boxcar apodization (top) and Hanning apodization (bottom). 
[6] L. J. Cutrona, W. E. Vivian, E. N. Leith, and G. O. Hall, "A high resolution radar combat-surveillance system," IRE Trans. Mil. Elect. MIL-5(2), pp. 127-131, 1961.

[7] C. W. Sherwin, J. P. Ruina, and D. Rawcliffe, "Some early developements in synthetic aperture radar systems," IRE Trans. Mil. Elect. MIL-6(2), pp. 111-115, 1962.

[8] J. C. Curlander and R. N. McDonough, Synthetic Aperture Radar: Systems and Signal Processing, John Wiley \& Sons, Inc., 1991.

[9] R. Thomson, "Transverse and longitudinal resolution of the synthetic aperture focusing technique," Ultrasonics 22, pp. 9-15, 1984.

[10] M. Karaman, P. C. Li, and M. O'Donnell, "Synthetic aperture imaging for small scale systems," IEEE Trans. Ultrason., Ferroelec., Freq. Contr. 42, pp. 429-442, 1995.

[11] C. Passmann and H. Ermert, "A 100-MHz ultrasound imaging system for dermatologic and ophthalmologic diagnostics," IEEE Trans. Ultrason., Ferroelec., Freq. Contr. 43, pp. 545-552, 1996.

[12] C. H. Frazier and W. D. O'Brien, "Synthetic aperture techniques with a virtual source element," IEEE Trans. Ultrason., Ferroelec., Freq. Contr. 45, pp. 196-207, 1998.

[13] S. I. Nikolov and J. A. Jensen, "Virtual ultrasound sources in high-resolution ultrasound imaging," in Proc. SPIE - Progress in biomedical optics and imaging, 3, pp. 395-405, 2002.

[14] S. I. Nikolov and J. A. Jensen, "3D synthetic aperture imaging using a virtual source element in the elevation plane," in Proc. IEEE Ultrason. Symp., 2, pp. 1743-1747, 2000.

[15] M. H. Bae and M. K. Jeong, "A study of synthetic-aperture imaging with virtual source elements in B-mode ultrasound imaging systems," in IEEE Trans. Ultrason., Ferroelec., Freq. Contr., 47, pp. 1510-1519, 2000.

[16] M. Bae, H. Lee, S. B. Park, R. Yoon, M. H. Jeong, D. G. Kim, M. Jeong, and Y. Kim, "A new ultrasonic synthetic aperture tissue harmonic imaging system," in Proc. IEEE Ultrason. Symp., pp. 1258-1261, 2008.

[17] J. Kortbek, J. A. Jensen, and K. L. Gammelmark, "Synthetic aperture sequential beamforming," in Proc. IEEE Ultrason. Symp., pp. 966-969, 2008.

[18] J. A. Jensen, J. Kortbek, S. I. Nikolov, M. C. Hemmsen, and B. Tomov, "Implementation of synthetic aperture imaging in medical ultrasound: The dual stage beamformer approach," in EUSAR, 2010.

[19] M. C. Hemmsen, J. M. Hansen, and J. A. Jensen, "Synthetic Aperture Sequential Beamformation applied to medical imaging using a multi element convex array transducer," in EUSAR, p. Accepted for publication, Apr. 2012.

[20] Y. Du, J. Rasmussen, H. Jensen, and J. A. Jensen, "Second harmonic imaging using synthetic aperture sequential beamforming," in Proc. IEEE Ultrason. Symp., pp. 2261-2264, 2011.

[21] J. H. Rasmussen, M. C. Hemmsen, S. S. Madsen, P. M. Hansen, M. B. Nielsen, and J. A. Jensen, "Implementation of tissue harmonic synthetic aperture imaging on a commercial ultrasound system," in Proc. IEEE Ultrason. Symp., p. Accepted, 2012.

[22] J. M. Hansen, M. C. Hemmsen, and J. A. Jensen, "An object-oriented multi-threaded software beamformation toolbox," in Proc. SPIE Med. Imag., 7968, p. 79680Y, March 2011. Lake Buena Vista (Orlando), Florida, USA.

[23] M. C. Hemmsen, M. M. Petersen, S. I. Nikolov, M. B., Nielsen, and J. A. Jensen, "Ultrasound image quality assessment: A framework for evaluation of clinical image quality," in Proc. SPIE Med. Imag., 76291, pp. 76290C-12, Medical Imaging 2010: Ultrasonic Imaging, Tomography, and Therapy, 2010.

[24] J. A. Jensen and N. B. Svendsen, "Calculation of Pressure Fields from Arbitrarily Shaped, Apodized, and Excited Ultrasound Transducers," IEEE Trans. Ultrason., Ferroelec., Freq. Contr. 39, pp. 262-267, 1992.

[25] J. A. Jensen, "Field: A program for simulating ultrasound systems," Med. Biol. Eng. Comp. 10th NordicBaltic Conference on Biomedical Imaging, Vol. 4, Supplement 1, Part 1, pp. 351-353, 1996. 